\title{
Prevalence of polycystic ovary syndrome in a sample of Indigenous women in Darwin, Australia
}

Jacqueline A Boyle FRANZCOG, MPH\&TM, PhD Senior Research Fellow

Joan Cunningham ScD, Senior Principal Research Fellow

Kerin O'Dea $\mathrm{PhD}$ Director

Terry Dunbar BBus, MProfEdTraining PhD Candidate. Senior Research Fellow and Director

Robert J Norman MD, FRANZCOG, FRCPA Director

1 Jean Hailes for Women's Health, Monash University Melbourne, VIC

2 Menzies School of Health Research, Darwin, NT.

3 The Robinson Institute University of Adelaide Adelaide, SA

4 The Sansom Institute University of South Australia, Adelaide, SA

5 Australian Centre for Indigenous Knowledges and Education

Charles Darwin University Darwin, NT.

jacqueline.boyle@ monash.edu

MJA 2012; 196: 62-66 doi: 10.5694/mjall.10553 olycystic ovary syndrome (PCOS) is the most common endocrine problem in women of reproductive age. ${ }^{1}$ The diagnosis is generally based on three components: (i) ovarian dysfunction, as manifested by oligomenorrhoea or anovulation; (ii) polycystic ovaries on ultrasound examination; and (iii) evidence of hyperandrogenism, either biochemical or clinical. ${ }^{2}$ The National Institutes of Health (NIH) 1990 definition, one of the most widely used, includes two of these, namely hyperandrogenaemia and anovulatory menstrual cycles, and exclusion of other aetiologies. ${ }^{3}$ The Rotterdam consensus (2003) recommends any two of the three components listed above, as well as exclusion of other aetiologies. ${ }^{2}$ These criteria for diagnosis of PCOS are listed in Box 1.

PCOS may cause difficulties in reproductive health with irregular menstrual cycles, anovulation and infertility, and is associated with hyperinsulinaemia and insulin resistance, impaired glucose tolerance, diabetes, dyslipidaemia and cardiovascular disease (CVD). ${ }^{1}$

The prevalence of PCOS is generally reported as $4 \%-8 \%$ of women of reproductive age, regardless of ethnicity. This range is based largely on three studies - in Spain, the United States and Greece - all of which used $\mathrm{NIH}$ criteria for diagnosing PCOS. ${ }^{4-6}$ Most women in these studies were of European origin, although the American study also included African Americans. ${ }^{5}$ Studies of retrospective birth cohorts in Finland and Australia, also using the NIH criteria, showed prevalences of $10.3 \%$ and $8.7 \%$, respectively. 7,8

There is some evidence that the prevalence of PCOS may be higher in populations with increased risks of insulin resistance and metabolic disease (eg, PCOS was found in $13 \%$ of Mexican-American women whose parents had a history of CVD). ${ }^{9}$ Many factors associated with PCOS are

Objective: To document the prevalence of polycystic ovary syndrome (PCOS) and its associated characteristics in a sample of urban Indigenous women.

Design: A cross-sectional survey of Indigenous women, including biochemical and anthropometric assessments. PCOS was assessed using the National Institutes of Health 1990 criteria.

Setting and participants: Indigenous women, aged 15-44 years, living in a defined area in and around Darwin, Northern Territory, Australia, September 2003 - March 2005.

Main outcome measures: Proportion of participants with PCOS overall and measures of obesity.

Results: Among 248 women eligible for assessment, the proportion who had PCOS was $15.3 \%$ (95\% CI, 10.8\%-19.8\%). The proportion with PCOS was similar across age groups, but was significantly higher $(P=0.001)$ in women with a body mass index (BMI) of $\geqslant 30.0 \mathrm{~kg} / \mathrm{m}^{2}(30.5 \%)$ compared with women with a BMI of $25.0-29.9 \mathrm{~kg} / \mathrm{m}^{2}(8.2 \%)$ or a BMl of $<25.0 \mathrm{~kg} / \mathrm{m}^{2}(7.0 \%)$.

Conclusions: A high proportion of these Indigenous women had PCOS. The significant relationship with obesity gives a strong rationale for screening for PCOS during routine care of Indigenous women who are obese and of reproductive age.

more common among Australian Indigenous women, including obesity, hyperinsulinism, diabetes, dyslipidaemia and a history of low birthweight. $^{10,11}$ One small study of 35 Australian Aboriginal women found a PCOS prevalence of $18 \%,{ }^{12}$ suggesting that PCOS may be more common in Australian Indigenous women than in other populations. Our study assessed the prevalence of PCOS, and the characteristics of women with and without PCOS, in a group of urban Indigenous women in Darwin, Northern Territory, Australia.

\section{Methods}

\section{Study population and design}

Data for our study were collected from September 2003 to March 2005 as part of the Darwin Region Urban Indigenous Diabetes (DRUID) study, which has been described in detail elsewhere. ${ }^{13}$ Briefly, eligible DRUID participants were volunteers aged $\geqslant 15$ years who identified as Aboriginal and/or Torres Strait Islander, had lived in a defined geographical region in the Darwin area for $\geqslant 6$ months, and were not living in an institution. Those who gave consent underwent a health examination, including collection of blood and urine samples and clinical and anthropometric measurements, and completed a questionnaire.

Our study was limited to female DRUID study participants who were aged 15-44 years, and who agreed to complete a questionnaire on women's reproductive health and/or to have additional analyses of their blood samples for androgen levels.

\section{Women's reproductive health questionnaire}

Information was collected on menstrual cycle frequency and regularity, fertility and infertility, menopause, past and present contraceptive use, and presence and treatment of hirsutism and acne. Oligomenorrhoea was defined as self-reported cycles $>35$ days apart, regardless of whether they were described as regular or irregular.

\section{Anthropometry and blood pressure}

The method of obtaining anthropometric measures (weight, height, body mass index [BMI] and waist : hip ratio [WHR]) has been described previously. ${ }^{13}$ BMI categories were defined as healthy $\left(<25.0 \mathrm{~kg} / \mathrm{m}^{2}\right)$, overweight $\left(\geqslant 25.0 \mathrm{~kg} / \mathrm{m}^{2}\right.$ and $\left.<30.0 \mathrm{~kg} / \mathrm{m}^{2}\right)$ and 
obese $\left(\geqslant 30.0 \mathrm{~kg} / \mathrm{m}^{2}\right)$. WHR categories were healthy $(<0.8)$ and overweight/ obese $(\geqslant 0.8)$. Blood pressure was measured in the sitting position after $5 \mathrm{~min}$ at rest using an automated Welch Allyn Spot Vital Signs monitor (Welch Allyn Medical Products, Skaneateles Falls, NY, USA).

\section{Assays}

Venepuncture was performed after an overnight fast. Sex hormone-binding globulin (SHBG) and androgen levels were analysed by the Reproductive Medicine Laboratory (RML) in Adelaide. SHBG was measured with an immunoradiometric assay (IRMA) (Orion Diagnostica [IRMA Cat. No. 68563], Espoo, Finland); testosterone was measured with a radioimmunoassay (RIA) kit (DSL-4100, Diagnostic System Laboratories, Webster, Tex, USA); and anti-Müllerian hormone $(\mathrm{AMH})$ levels were measured using a commercially available $\mathrm{AMH}$ enzyme immunoassay (Beckman Coulter, Marseille, France). Free androgen index (FAI) was calculated as: total testosterone (nmol/L) / SHBG $(\mathrm{nmol} / \mathrm{L}) \times 10$; and free testosterone was calculated using the Vermeulen equation, assuming a serum albumin concentration of $43 \mathrm{~g} / \mathrm{L}^{14}$

To exclude other causes of hyperandrogenaemia and oligomenorrhoea, apart from PCOS, women with an FAI of $>5.4$ plus oligomenorrhoea had further analysis of their samples for levels of 17-hydroxyprogesterone (17$\mathrm{OHP})$, dehydroepiandrosterone sulfate (DHEAS), thyroid-stimulating hormone (TSH), and prolactin. The FAI cut-off of 5.4, chosen at the study inception, was the upper limit of normal for women tested at the RML.

The RML also performed these analyses: 17-OHP estimation with an RIA kit (DSL-6800) and DHEAS level using the DHEAS RIA kit (DSL-2700) (Diagnostic System Laboratories); and TSH and prolactin levels using the ADVIA Centaur automated chemiluminescence immunoassay system (Bayer HealthCare, Australia).

Inter- and intra-assay coefficients of all tests were $<10 \%$.

\section{Exclusion criteria}

Excluded from assessment of PCOS (Box 2) were women who were pregnant or breastfeeding; those who did

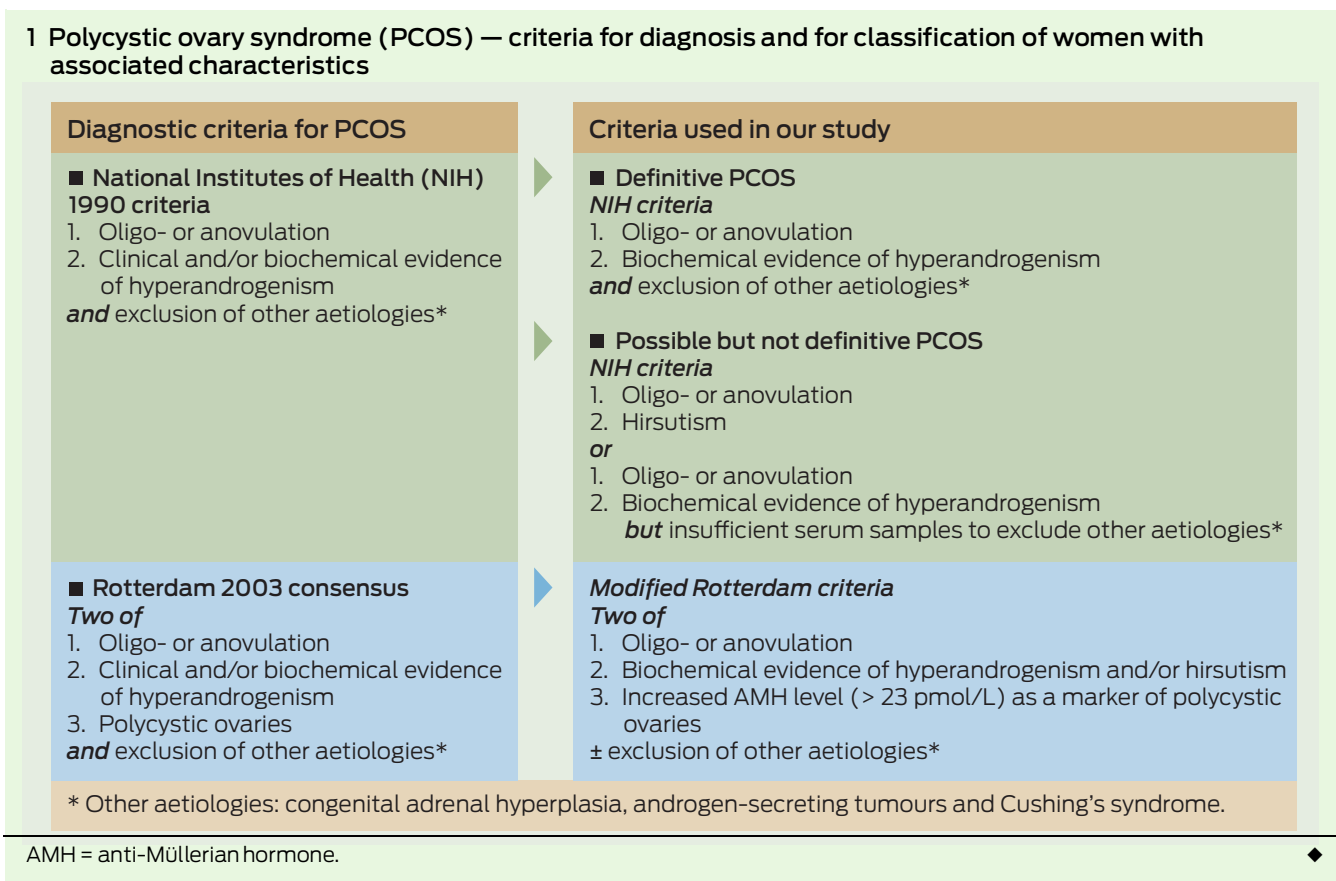

not consent to complete a reproductive health questionnaire; those without androgen level assessment; those who reported being menopausal, using hormone replacement therapy or hormonal contraception, or who had had a hysterectomy or oophorectomy (they may have had difficulty recalling menstrual cycle regularity); and those with missing information about cycle regularity.

\section{Classification of PCOS and associated categories (Box 1 )}

\section{a) PCOS-NIH}

PCOS was defined using NIH 1990 criteria. Information was available on the presence of (i) self-reported menstrual dysfunction; (ii) hyperandrogenaemia; and (iii) exclusion of other disorders. ${ }^{3}$ Menstrual dysfunction was considered present for women with oligomenorrhoea. Hyperandrogenaemia was defined as a circulating free testosterone level $>34.2 \mathrm{pmol} / \mathrm{L}$ (the 95th percentile for a group of women known to be free of PCOS whose samples were assessed on the same machine with the same assay as our study). Although hirsutism was selfreported as excess hair on the chin, lip, chest or abdomen, this was not used as a diagnostic criterion because it was not assessed objectively by a trained rater.

Women were included in the PCOS-NIH group if they had: (i) oli- gomenorrhoea; (ii) a free testosterone level of $>34.2 \mathrm{pmol} / \mathrm{L}$; and (iii) normal values for prolactin, $17-\mathrm{OHP}$ and TSH.

\section{b) Abnormal prolactin, 17-OHP and TSH values}

Women with oligomenorrhoea and elevated free testosterone levels were excluded from a diagnosis of PCOS if they had abnormal values for prolactin (>900 IU/L), 17-OHP (>10 mmol/L), and TSH $(<0.4$ or $>4.7 \mathrm{mIU} / \mathrm{L})$.

\section{c) Possible but not definitive PCOS}

Women were included in the "possible but not definitive" group if they were not in either group (a) or (b) above and had: (i) self-reported hirsutism plus oligomenorrhoea (possible PCOS-NIH); (ii) the two $\mathrm{NIH}$ components of PCOS (oligomenorrhoea and hyperandrogenaemia), but were missing measurements of 17$\mathrm{OHP}$, prolactin or TSH and, therefore, were unable to have abnormalities of these excluded; or (iii) possible PCOS by modified Rotterdam criteria of two components of hyperandrogenaemia or hirsutism, oligomenorrhoea and a high $\mathrm{AMH}$ level. Women in this latter group with completed investigations (TSH, prolactin and 17-OHP) have PCOS by these criteria.

AMH level was used as a surrogate for antral follicle count, as described by Pigny et al. ${ }^{15}$ An AMH cut-off of $23.0 \mathrm{pmol} / \mathrm{L}$ was deemed 
to be consistent with the presence of polycystic ovaries, based on a study of women in Adelaide with and without PCOS. This level was shown to have a sensitivity of $75 \%$ and a specificity of $90 \%$ in diagnosing polycystic ovaries confirmed by ultrasound examination. ${ }^{16}$ However, in our study, correlating $\mathrm{AMH}$ levels with ultrasound results was not feasible; these women are included as having possible PCOS by the Rotterdam criteria, ${ }^{2}$ but no further analysis of this group is reported here.

\section{d) Non-hyperandrogenic}

This group includes women with no hyperandrogenaemia or self-reported hirsutism and an AMH level of $<23.0 \mathrm{pmol} / \mathrm{L}$. They had either regular cycles (<35 days) or oligomenorrhoea.

\section{e) Hyperandrogenaemia}

These women had free testosterone levels of $>34.2 \mathrm{pmol} / \mathrm{L}$, regular cycles of $<35$ days, and an AMH level of $<23.0 \mathrm{pmol} / \mathrm{L}$.

\section{f) Idiopathic hirsutism}

These women had self-reported hirsutism, but normal androgen levels (free testosterone level, $<34.2 \mathrm{pmol} / \mathrm{L}$ ), regular cycles of $<35$ days, and an AMH level of $<23.0 \mathrm{pmol} / \mathrm{L}$.

\section{Statistical analysis}

Statistical analyses were performed using Intercooled Stata 8.0 (StataCorp, College Station, Tex, USA). Frequencies, means, medians and SDs were calculated, and data are presented as median (interquartile range [IQR]). Overall differences across the groups were compared using a Kruskal-Wallis test and post-hoc pairwise comparisons were made using a Mann-Whitney rank-sum test. A two-tailed $\chi^{2}$ test was used to compare categorical variables.

\section{Ethics approval}

Our study was approved by the Human Research Ethics Committee of the Northern Territory Department of Health and Menzies School of Health Research, including its Aboriginal Ethics Sub-Committee. Our study received guidance from an Indigenous steering committee of local community leaders.

2 Selection of study participants for assessment of prevalence of polycystic ovary syndrome

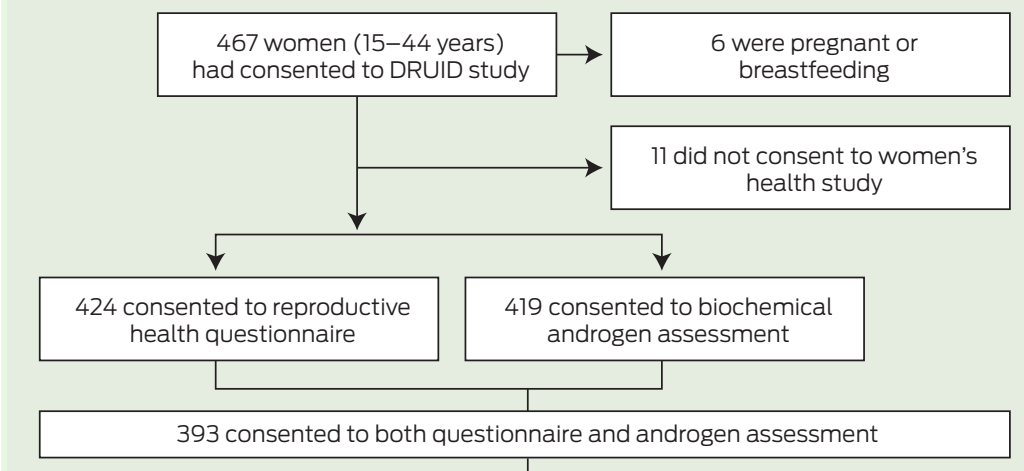

Taking current hormonal contraception or hormone replacement therapy, or reported hysterectomy or menopause

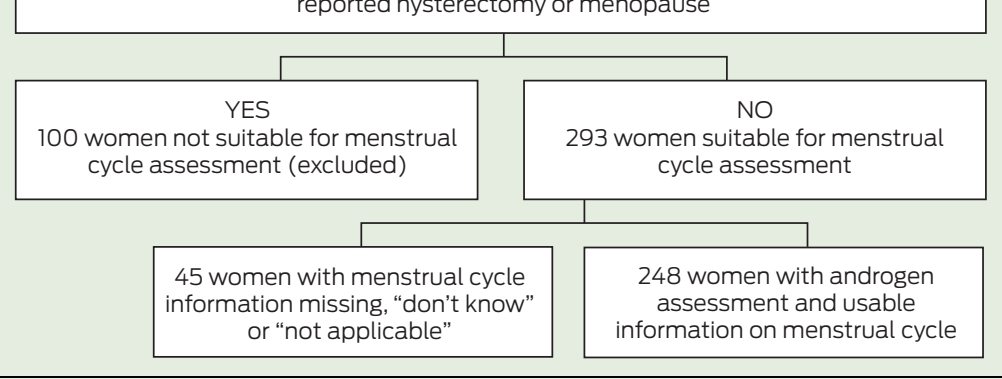

DRUID = Darwin Region Urban Indigenous Diabetes study.

\section{Results}

The selection of study participants is shown in Box 2. Of 461 women, 424 $(92.0 \%)$ answered the questionnaire, 419 had androgen levels measured (90.9\%), and $393(85.2 \%)$ completed both the questionnaire and had androgen levels measured. Based on estimated resident population figures at June $2004,{ }^{17}$ our participants represent at least $13 \%-14 \%$ of the eligible women in this age group living in a defined region of Darwin.

Of these 393 women, 248 fulfilled all protocol criteria and were eligible for assessment of PCOS (Box 3). The median age of this group was 31.0 years (IQR, 22.0-38.0 years), median BMI was $27.0 \mathrm{~kg} / \mathrm{m}^{2}$ (IQR, 22.5$\left.32.6 \mathrm{~kg} / \mathrm{m}^{2}\right)$ and median WHR was 0.85 (IQR, 0.80-0.91).

Thirty-eight of these women (15.3\%; 95\% CI, 10.8\%-19.8\%) met the diagnosis of PCOS by NIH criteria. The proportion of women with PCOS increased with increasing BMI $(P=0.001)$ from $7.0 \%(7 / 100)$ of the "healthy" group, to $8.2 \%$ (5/61) of the "overweight" group and 30.5\% (25/82) of the "obese" group. By contrast, there was no significant difference in the proportion of women with PCOS between the healthy and overweight categories according to WHR ( $P=$ $0.61)$ : healthy $13.4 \%(9 / 67)$ versus overweight $16.0 \%$ (29/181).

The remaining women were categorised as shown in Box 3: hyperandrogenaemia, 34 (13.7\%); idiopathic hirsutism, 25 (10.1\%); non-hyperandrogenic, 79 (31.9\%); abnormal 17OHP/TSH values, 9 (3.6\%); and possible but not definitive PCOS, 61 (24.6\%). The majority of the latter group $(n=53)$ had possible PCOS by the modified Rotterdam criteria. Two additional women with insufficient serum for measurement of AMH level (one with a free testosterone level of $>34.2 \mathrm{pmol} / \mathrm{L}$, regular cycles and hirsutism, and the other with hirsutism only) could not be allocated to the above groups.

The characteristics of women in four of these groups (PCOS, hyperandrogenaemia, idiopathic hirsutism and non-hyperandrogenic) are reported in Box 4. Median age was similar across the non-hyperandrogenic, hyperandrogenaemia and PCOS groups, but those with idiopathic hirsutism were significantly older. Women with PCOS had the highest median BMI $\left(33.4 \mathrm{~kg} / \mathrm{m}^{2}\right)$ and the highest systolic and diastolic blood pressures. Total testosterone and free testosterone levels were 
highest and SHBG levels lowest in women with PCOS and in those with hyperandrogenaemia.

The proportion of women with diabetes was not significantly different across these four groups $(P=0.12)$; eight women had known diabetes before participating in the DRUID study and three in each of the nonhyperandrogenic group and the PCOS group were newly diagnosed with diabetes during our study. No significant differences were observed in fasting glucose levels between the groups $(P=0.19)$.

\section{Discussion}

Using the NIH 1990 criteria for diagnosis, PCOS was present in around one in six $(15.3 \%)$ urban Indigenous women volunteering for our study in Darwin. This proportion increased with increasing BMI, reaching 30.5\% in those with a BMI of $>30.0 \mathrm{~kg} / \mathrm{m}^{2}$. Conversely, there was no significant difference in the proportion of women with PCOS with increasing WHR. Given that Indigenous Australians (both men and women) when they gain weight have a centralised (abdominal) pattern of fat distribution, an elevated WHR is not as good a discriminator of increasing overweight and obesity as $\mathrm{BMI}$ is. ${ }^{18}$

The prevalence of PCOS in our study group is higher than reported in studies in other countries also using $\mathrm{NIH}$ criteria - Spain $(6.5 \%),{ }^{4}$ the US $(4.0 \%),{ }^{5}$ Greece $(6.8 \%),{ }^{6}$ and northern Finland (10.3\%). ${ }^{7}$ Another Australian study in a non-Indigenous population found a proportion of $8.7 \%,{ }^{8}$ and a

3 Classification of eligible participants for assessment of prevalence of polycystic ovary syndrome (PCOS)

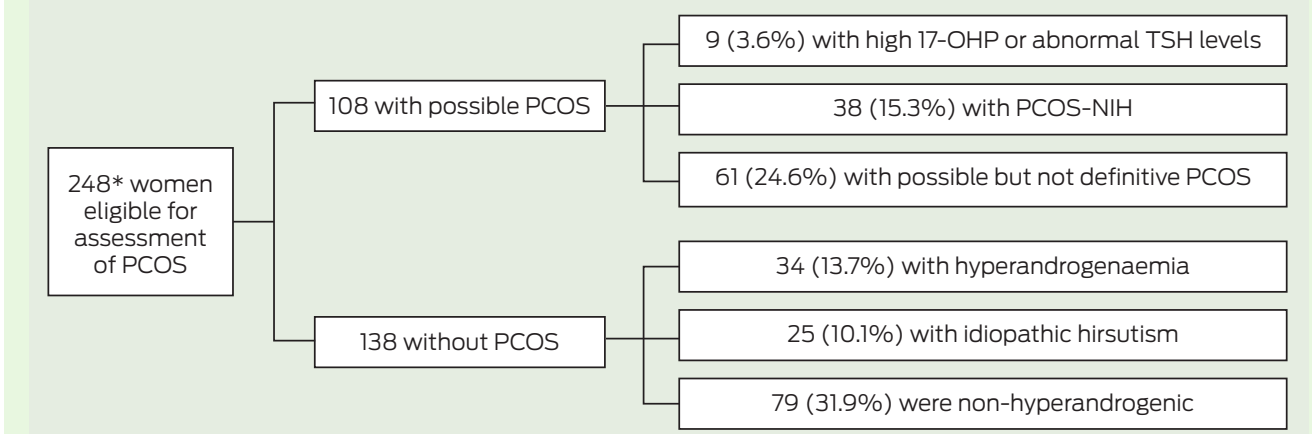

17-OHP = 17-hydroxyprogesterone. TSH = thyroid-stimulating hormone. NIH = National Institutes of Health. * Two women omitted, as insufficient serum was available for measuring anti-Müllerian hormone levels.

study of Mexican-Americans found that $13 \%$ had PCOS. $^{9}$ However, differences in study populations and definitions of PCOS make it difficult to compare these studies. In our study, the proportion of women with PCOS would almost certainly have been higher using a different definition, given that at least 14 women (5.6\%) who did not meet the NIH definition had PCOS according to the modified Rotterdam criteria described.

Recruitment strategies have varied among the prevalence studies, from population-based to employmentbased to volunteers for screening. The women in our study were community-based volunteers, but the larger DRUID study in which they also participated focused on diabetes screening. Therefore, women at high risk of diabetes, or who were obese, may have been more likely to participate. Of the total number of women aged 15-44 years in the DRUID study, 8.3\% had known or newly diagnosed dia- betes. Age-specific figures (2.9\% among 15-24 years, $8.8 \%$ among 2534 years and $13.8 \%$ among $35-44$ years) were only slightly higher than those self-reported for known diabetes or high blood sugar levels in the National Aboriginal and Torres Strait Islander health survey (2004-05), ${ }^{19}$ which does not support an excess of high-risk women selecting for screening. Similarly, while $33.7 \%$ of women in our study were obese (BMI $>30.0 \mathrm{~kg} / \mathrm{m}^{2}$ ), this is consistent with national data, and the median BMI in our study was similar to that for women in other PCOS prevalence studies. ${ }^{5,8}$

Although the PCOS prevalence we found is higher than that in previous reports, the constraints of our study suggest this prevalence may even be an underestimate. Previously reported prevalence studies have used a number of androgen measurements to determine hyperandrogenaemia, whereas the definition used for hyperandrogenaemia in our study

4 Characteristics of women (median [interquartile range]) by classification - non-hyperandrogenic, idiopathic hirsutism, hyperandrogenaemia, and polycystic ovary syndrome - National Institutes of Health criteria (PCOS-NIH)

\begin{tabular}{|c|c|c|c|c|c|}
\hline Characteristics & $\begin{array}{c}\text { Non-hyperandrogenic } \\
\qquad(n=79)\end{array}$ & $\begin{array}{l}\text { Idiopathic hirsutism } \\
\qquad(n=25)\end{array}$ & $\begin{array}{l}\text { Hyperandrogenaemia and } \\
\text { regular cycles }(n=34)\end{array}$ & PCOS-NIH $(n=38)$ & $\begin{array}{c}P \text { value } \\
\text { (Kruskal-Wallis) }\end{array}$ \\
\hline Age (years) & $33.0(23.0-39.0)$ & $37.0(34.0-40.0) *^{\dagger}$ & $32.0(23.0-38.0)^{\ddagger}$ & $31.5(21.0-36.0)$ & 0.08 \\
\hline Body mass index $\left(\mathrm{kg} / \mathrm{m}^{2}\right)$ & $23.7(20.7-27.6) *$ & $27.4(23.0-33.4) *$ & $28.1(25.5-34.2) *$ & $33.4(27.7-39.7)$ & 0.001 \\
\hline Waist: hip ratio & $0.83(0.79-0.90)^{* \ddagger}$ & $0.87(0.81-0.95)$ & $0.85(0.82-0.90)$ & $0.86(0.82-0.96)$ & 0.05 \\
\hline Systolic BP (mmHg) & $106.0(100.0-114.0) *$ & $106.5(101.5-121.0) *$ & $106.8(101.5-113.0)$ & $112.3(105.0-118.5)$ & 0.06 \\
\hline Diastolic BP (mmHg) & $69.5(65.0-74.0) *$ & $72.0(66.0-77.5)$ & $71.0(63.5-77.0)$ & $73.8(67.0-80.0)$ & 0.06 \\
\hline Total testosterone (nmol/L) & $1.3(1.1-1.6) * \ddagger$ & $1.3(1.2-1.5) *$ & $2.4(1.9-2.6)^{\ddagger}$ & $2.3(1.9-3.0)$ & 0.001 \\
\hline Sex hormone-binding globulin ( $\mathrm{nmol} / \mathrm{L}$ ) & $41.6(30.2-63.0) *$ & $40.8(24.7-46.6) *$ & $20.8(17.4-27.4)$ & $19.4(13.7-25.4)$ & 0.001 \\
\hline Free testosterone (pmol/L) & $21.0(13.8-27.3)^{* \ddagger}$ & $23.7(15.0-30.9)^{*}$ & $50.2(44.0-60.0)^{\ddagger}$ & $50.9(41.0-78.5)$ & 0.001 \\
\hline Anti-Müllerian hormone (pmol/L) & $14.8(9.9-26.1)$ & $10.3(7.9-17.2)$ & $12.2(8.6-18.7)$ & $29.9(11.1-44.9)$ & 0.001 \\
\hline Fasting glucose (mmol/L) & $4.9(4.7-5.4)$ & $5.3(4.8-5.5)$ & $5.1(4.9-5.4)$ & $5.1(4.8-5.7)$ & 0.19 \\
\hline Diabetes, no. (\%) & $4(5.1 \%)$ & $4(16.0 \%)$ & $1(2.9 \%)$ & $5(13.2 \%)$ & 0.12 \\
\hline
\end{tabular}

$\mathrm{BP}=$ blood pressure. $P$ values based on pair-wise comparisons using a Mann-Whitney rank-sum test. $* P<0.05$ compared with $\mathrm{PCOS}$ group; $\uparrow P<0.05$ compared with non-hyperandrogenic group; $\ddagger P<0.05$ compared with idiopathic hirsutism group. 
only included free testosterone level; this may have led to an underestimation of prevalence. Finally, excluding women using hormonal contraception may have biased the estimation either way, with the direction depending on the reason for use. If hormonal contraception was used for cycle control then it would likely have underestimated the true prevalence of PCOS. However, no information was available on reasons for contraceptive use.

Hirsutism, including self-reported hirsutism, has been included as diagnostic of hyperandrogenism in PCOS in a number of major prevalence studies. ${ }^{4,5,7-9}$ We did not use it as a diagnostic factor for PCOS in our study; however, $38 \%$ of women reported hair growth on their lip, chin or chest. Previous studies found up to $82 \%$ of women presenting with hirsutism have PCOS, even those with a low Ferriman-Gallwey score; ${ }^{20}$ thus excluding hirsutism probably underestimated PCOS prevalence. Compared with studies of women of European origin, a higher proportion of women in our study reported hirsutism, However, hirsutism varies with ethnicity, and these figures are consistent with reports in other Australian Indigenous women (39.5\%). ${ }^{12}$

The high prevalence of PCOS found in our sample may be related to the high risk of diabetes and obesity in Australian Indigenous women, as well as antenatal factors (low birthweight and diabetes in pregnancy) or environmental factors (eg, diet, physical activity and smoking) that have an impact on insulin sensitivity and hormones such as testosterone and SHBG. Community and health care providers need to be aware of both diagnosis and best-practice manage- ment of $\mathrm{PCOS}^{21}$ so that there is a low threshold for screening Indigenous women for PCOS.

Acknowledgements: We gratefully acknowledge the support of DRUID study participants, study staff, members of the Indigenous Steering Group, and partner organisations. The DRUID Study was funded by the National Health and Medical Research Council (Project Grant \#236207), with additional support from the Australian Government Department of Employment and Workplace Relations, the Clive and Vera Ramaciotti Foundation, the Vincent Fairfax Family Foundation, the AusDiab Partnership in Type 2 Diabetes, and Bayer HealthCare. The DRUID study is an in-kind project of the Cooperative Research Centre for Aboriginal Health.

Jacqueline Boyle was supported by Royal Australian and New Zealand College of Obstetricians and Gynaecologists Fotheringham Research Grant, 2002; NHMRC Training Scholarship for Indigenous Australian Health Research, 2005-2009; Australian Academy of Science Douglas and Lola Douglas Scholarship, 2005-2009; and Eli Lily. Joan Cunningham was supported by an NHMRC Career Development Award (No. 283310) and NHMRC Research Fellowship (No. 545200)

Competing interests: No relevant disclosures.

Received 2 May 2011, accepted 11 Oct 2011.

1 Norman RJ, Dewailly D, Legro RS, Hickey TE. Polycystic ovary syndrome. Lancet 2007; 370: 685-697.

2 RotterdamESHRE/ASRM-Sponsored PCOS Consensus Workshop Group. Revised 2003 consensus on diagnostic criteria and long-term health risks related to polycystic ovary syndrome. Fertil Steril 2004; 81: 19-25.

3 Zawadzki JK, Dunaif A. Diagnostic criteria for polycystic ovary syndrome. In: Dunaif A, Givens JR, Haseltine F, editors. Polycystic ovary syndrome. Boston: Blackwell Scientific, 1992: 377-384.

4 Asuncion M, Calvo RM, San Millan JL, et al. A prospective study of the prevalence of the polycystic ovary syndrome in unselected Caucasian women from Spain. J Clin Endocrinol Metab 2000; 85: 2434-2438.

5 Knochenhauer ES, Key TJ, Kahsar-Miller M, et al. Prevalence of the polycystic ovary syndrome in unselected black and white women of the south eastern United States: a prospective study. J Clin Endocrinol Metab 1998; 83: 3078-3082.

6 Diamanti-Kandarakis E, Kouli CR, Bergiele AT, et al. A survey of the polycystic ovary syndrome in the Greek island of Lesbos: hormonal and metabolic profile. J Clin Endocrinol Metab 1999; 84: 4006-4119.

7 Taponen S, Martikainen H, Järvelin MR, et al. Metabolic cardiovascular disease risk factors in women with self-reported symptoms of oligomenorrhea and/or hirsutism: Northern Finland Birth Cohort 1966 Study. J Clin Endocrinol Metab 2004; 89: 2114-2118.

8 March WA, Moore VM, Willson KJ, et al. The prevalence of polycystic ovary syndrome in a community sample under contrasting diagnostic criteria. Hum Reprod 2010; 25: 544-551.

9 Goodarzi MO, Quinones MJ, Azziz R, et al. Polycystic ovary syndrome in MexicanAmericans: prevalence and association with the severity of insulin resistance. Fertil Steril 2005; 84: 766-912.

10 Vos T, Barker B, Stanley L, Lopez AO. The burden of disease and injury in Aboriginal and Torres Strait Islander peoples 2003. Brisbane: School of Population Health, University of Queensland, 2007.

11 Laws PJ, Hilder L. Australia's mothers and babies 2006. Sydney: Australian Institute of Health and Welfare National Perinatal Statistics Unit, 2008. (AlHWCat. No. PER 46; Perinatal Statistics Series No. 22.)

12 Davis SR, Knight S, White V, et al. Preliminary indication of a high prevalence of polycystic ovary syndrome in Indigenous Australian women. Gynecol Endocrinol 2002; 16: 443-446.

13 Cunningham J, O'Dea K, Dunbar T, et al. Study protocol - diabetes and related conditions in urban Indigenous people in the Darwin, Australia region: aims, methods and participation in the DRUID Study. BMC Public Health 2006; 6: 8.

14 Vermeulen A, Verdonck L, Kaufman JM. A critical evaluation of simple methods for the estimation of free testosterone in serum. J Clin Endocrinol Metab 1999: 84: 3666-3672.

15 Pigny P, Jonard S, Robert Y, Dewailly D. Serum anti-Mullerian hormone as a surrogate for antral follicle count for definition of the polycystic ovary syndrome. J Clin Endocrinol Metab 2006; 91: 941-945.

16 Poprzeczny A. Anti-Mullerian hormone as a diagnostic marker and pathogenic factor in polycystic ovary syndrome [Honours thesis]. Adelaide: University of Adelaide, 2007.

17 Australian Bureau of Statistics. Experimental projections of Aboriginal and Torres Strait Islander populations, ATSIC regions. Canberra: ABS, 2004. (ABS Cat. No. 3238.0.55.002.)

18 Piers LS, Rowley KG, Soares MJ, O'Dea K. Relation of adiposity and body fat distribution to body mass index in Australians of Aboriginal and European ancestry. Eur J Clin Nutr 2003; 57: 956-963.

19 Australian Bureau of Statistics. National Aboriginal and Torres Strait Islander Health Survey 2004-05. Canberra: ABS, 2006. (ABS Cat. No. 4715.0.) http://www.abs.gov.au/ausstats/ abs@.nsf/mf/4715.0 (accessed Oct 2011).

20 Azziz R, Sanchez LA, Knochenhaver ES, et al. Androgen excess in women: experience with over 1000 consecutive patients. J Clin Endocrinol Metab 2004; 89: 453-462.

21 Teede HJ, Misso ML, Deeks AA, et al; the Guideline Development Groups. Assessment and management of polycystic ovary syndrome: summary of an evidence-based guideline. Med J Aust 2011; 195 (6 Suppl): S65-S112.

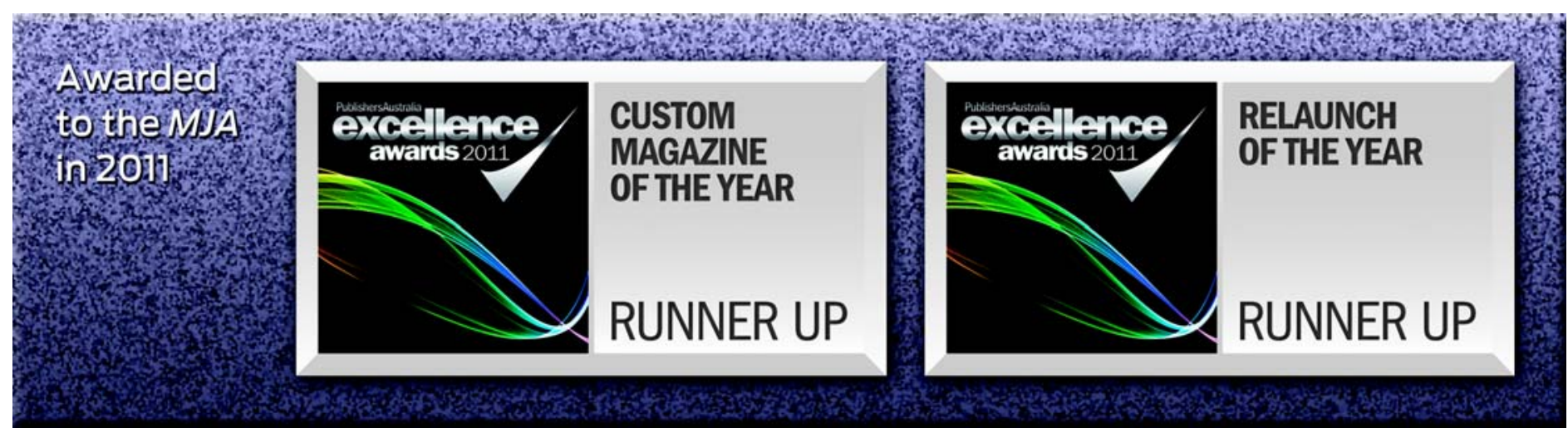

